\title{
Optimizing Different Loss Functions in Multilabel Classifications
}

\author{
Jorge Díez • Oscar Luaces • Juan José del Coz • Antonio Bahamonde
}

Received: date / Accepted: date

\begin{abstract}
Multilabel classification (ML) aims to assign a set of labels to an instance. This generalization of multiclass classification yields to the redefinition of loss functions and the learning tasks become harder. The objective of this paper is to gain insights into the relations of optimization aims and some of the most popular performance measures: subset (or 0/1), Hamming, and the example-based F-measure. To make a fair comparison, we implemented three ML learners for optimizing explicitly each one of these measures in a common framework. This can be done considering a subset of labels as a structured output. Then we use Structured output Support Vector Machines (SSVM) tailored to optimize a given loss function. The paper includes an exhaustive experimental comparison. The conclusion is that in most cases, the optimization of the Hamming loss produces the best or competitive scores. This is a practical result since the Hamming loss can be minimized using a bunch of binary classifiers, one for each label separately, and therefore it is a scalable and fast method to learn ML tasks. Additionally, we observe that in noise free learning tasks optimizing the subset loss is the best option, but the differences are very small. We have also noticed that the biggest room for improvement can be found when the goal is to optimize an F-measure in noisy learning tasks.
\end{abstract}

Keywords Multilabel Classification · Structured Outputs · Optimization · Tensor Product

J. Díez · O. Luaces · J.J. del Coz · A. Bahamonde

Artificial Intelligence Center

University of Oviedo

33204 - Gijón (Spain)

Tel.: +34985182588

E-mail: \{jdiez,oluaces,juanjo,abahamonde\}@uniovi.es

\section{Introduction}

The aim of multilabel classification (ML) is to obtain simultaneously a collection of binary classifications; the positive classes are referred to as labels, the so-called relevant labels of the instances. Many complex classification tasks can be included into this paradigm. In document categorization, items are tagged for future retrieval; frequently, news or other kind of documents should be annotated with more than one label according to different points of view. Other application fields include semantic annotation of images and video, functional genomics, music categorization into emotions and directed marketing. Tsoumakas et al. [26,27] have made a detailed presentation of ML and their applications.

A number of strategies to tackle multilabel classification tasks have been published. Basically, they can be divided in two groups. Strategies in the first group try to transform the learning tasks into a set of single-label (binary or multiclass) classification tasks. Binary Relevance (BR) is the simplest, but very effective, transformation strategy. Each label is classified as relevant or irrelevant without any relation with the other labels. On the other hand, genuine multilabel strategies try to take advantage of correlation or interdependence between labels. In other words, these strategies try to take into account that the relevance of a label is conditioned not only by the feature values of an instance, but also by the values of the remaining labels.

There are several alternatives to assess the loss of ML classifiers. Since the aim is to predict a subset, it is possible to count the number of times that the predicted and the true subsets are different. But this is probably a too severe way to measure the loss. To obtain more sensitive loss functions it is possible to assess the degree of coincidence of predictions and true sets using some 
measures drawn from the field of Information Retrieval. This is the case of Precision, Recall, and their harmonic averages: F-measures.

In the literature there are algorithms that try to learn using heuristic strategies to achieve a good performance, but the results are no guaranteed to fulfill the expectations. Other methods explicitly aim to optimize the scores provided by a given loss function. However, these methods are not easy to scale for learning tasks with many features or labels. The objective of this paper is to gain insight into the relations of optimization aims and loss functions. We suggest some guidelines to solve a tradeoff between algorithmic complexity and performance of multilabel learners.

In the following sections we present three learning algorithms specially devised to optimize specific loss functions considering ML classifiers as structured output functions. This framework leads us to a tensor space of input instances and subsets of labels. The advantage of this approach is that it provides a common framework that allows a fair comparison of scores.

The ML learners are built adapting a Joachims' algorithm devised for optimizing multivariate loss functions like F-measures [12]. In this context the first contribution of the paper is to present an efficient algorithm for optimizing loss functions based on contingency tables in ML learning tasks. The second contribution is an exhaustive empirical study devised to compare the relation of these learners and the loss functions under different levels of label noise.

The paper is organized as follows. The next section reviews a formal framework for ML learning tasks, hypotheses, and loss functions. The structured output approach is presented in Section 3. Then, we introduce the optimization algorithms devised for three different loss functions. Then a section is devoted to review the related work. An experimental comparison is reported in Section 6. The last section summarizes some conclusions.

\section{Formal Framework for Multilabel Classification}

Let $\mathscr{L}$ be a finite and non-empty set of labels $\left\{l_{1}, \ldots, l_{L}\right\}$, let $\mathcal{X}$ be an input space, and let $\mathcal{Y}$ be the output space, the set of subsets (power set) of labels. A multilabel classification task can be represented by a dataset

$D=\left\{\left(\boldsymbol{x}_{1}, \boldsymbol{y}_{1}\right), \ldots,\left(\boldsymbol{x}_{n}, \boldsymbol{y}_{n}\right)\right\} \subset \mathcal{X} \times \mathcal{Y}$

of pairs of input instances $\boldsymbol{x}_{i} \in \mathcal{X}$ and subsets of labels $\boldsymbol{y}_{i} \in \mathcal{Y}$.
We identify the output space $\mathcal{Y}$ with vectors of dimension $L$ with components in $\{0,1\}$.

$\mathcal{Y}=\mathscr{P}(\mathscr{L})=\{0,1\}^{L}$.

In this sense for $\boldsymbol{y} \in \mathcal{Y}$,

$l \in \boldsymbol{y} \quad$ and $\quad \boldsymbol{y}[l]=1$

will appear interchangeably and will be considered equivalent.

The goal of a multilabel classification task $D$ is to induce a ML hypothesis defined as a function $h$ from the input space to the output space,

$h: \mathcal{X} \longrightarrow \mathcal{Y}$.

\subsection{Loss Functions for Multilabel Classification}

ML classifiers can be evaluated from different points of view. The predictions can be considered as a bipartition or a ranking of the set of labels. In this paper the performance of ML classifiers will be evaluated as a bipartition. Thus, loss functions must compare subsets of labels.

Usually these measures can be divided in two groups [27]. The example-based measures compute the average differences of the actual and the predicted sets of labels over all examples.

The label-based measures decompose the evaluation into separate assessments for each label. There are two options here, averaging the measure labelwise (usually called macro-average), or computing a single value over all predictions, the so-called micro-average version of a measure. In a previous work [7] we proposed an approach to improve micro-averaged measures. Interestingly, the optimization of macro-averaged measures is equivalent to the optimization of those measures in the subordinate Binary Relevance (BR) classifiers.

The goal in this paper is to optimize example-based measures. For further reference, let us recall the formal definitions of these measures. For a prediction of a multilabel hypothesis $h(\boldsymbol{x})$ and a subset of truly relevant labels $\boldsymbol{y} \subset \mathscr{L}$ the most basic loss function is the $0 / 1$ loss (usually called subset $0 / 1$ loss). This loss function assesses value 0 when the prediction is the same as the true value, and 1 in other cases. In symbols,

$\Delta_{0 / 1}(\boldsymbol{y}, h(\boldsymbol{x}))=\llbracket \boldsymbol{y} \neq h(\boldsymbol{x}) \rrbracket$

where the value of $\llbracket q \rrbracket$ for a predicate $q$ is 1 when it is true, and 0 otherwise.

But this measure is too strict. In fact it is blind to appreciate the degree of the discrepancy between prediction and true values. The $0 / 1$ loss ignores if $h(\boldsymbol{x})$ and 
$\boldsymbol{y}$ differ in one or in all the labels. To introduce more sensitive measures, for the prediction $h(\boldsymbol{x})$ of each example $(\boldsymbol{x}, \boldsymbol{y})$ we can compute the following contingency matrix, where $l \in \mathscr{L}$ :

\begin{tabular}{c|cc} 
& $l \in \boldsymbol{y}$ & $l \notin \boldsymbol{y}$ \\
\hline$l \in h(\boldsymbol{x})$ & $a$ & $b$
\end{tabular}

$l \notin h(\boldsymbol{x}) \quad c \quad d$

in which each entry $(a, b, c, d)$ is the number of times that the corresponding combination of memberships occurs. Notice that $a+b+c+d=L$.

The example-based performance measures are now defined as follows. Throughout these definitions,

$D^{\prime}=\left\{\left(\boldsymbol{x}^{\prime}{ }_{1}, \boldsymbol{y}_{1}^{\prime}\right), \ldots,\left(\boldsymbol{x}_{n^{\prime}}^{\prime}, \boldsymbol{y}_{n^{\prime}}^{\prime}\right)\right\}$

is a test set.

Definition 1 The Recall is defined as the proportion of truly relevant labels that are included in predictions. The Recall for an input $\boldsymbol{x}^{\prime}$ and the average Recall are given by

$R\left(h\left(\boldsymbol{x}^{\prime}\right), \boldsymbol{y}^{\prime}\right)=\frac{a}{a+c} ; \quad R=\frac{\sum_{\boldsymbol{x}^{\prime} \in D^{\prime}} R\left(h\left(\boldsymbol{x}^{\prime}\right), \boldsymbol{y}^{\prime}\right)}{\left|D^{\prime}\right|}$.

Definition 2 The Precision is defined as the proportion of predicted labels that are truly relevant. The Precision for an input $\boldsymbol{x}^{\prime}$ and the average Precision are given by

$P\left(h\left(\boldsymbol{x}^{\prime}\right), \boldsymbol{y}^{\prime}\right)=\frac{a}{a+b} ; \quad P=\frac{\sum_{\boldsymbol{x}^{\prime} \in D^{\prime}} P\left(h\left(\boldsymbol{x}^{\prime}\right), \boldsymbol{y}^{\prime}\right)}{\left|D^{\prime}\right|}$.

The trade-off between Precision and Recall is formalized by their harmonic mean. So, in general, the $F_{\beta}$ $(\beta \geq 0)$ is defined by

$F_{\beta}=\frac{\left(1+\beta^{2}\right) P \cdot R}{\beta^{2} P+R}$.

Definition 3 The $F_{\beta}(\beta \geq 0)$ for an example and the average values of a test set are defined by

$$
\begin{aligned}
F_{\beta}\left(h\left(\boldsymbol{x}^{\prime}\right), \boldsymbol{y}^{\prime}\right) & =\frac{\left(1+\beta^{2}\right) a}{\left(1+\beta^{2}\right) a+b+\beta^{2} c} ; \\
F_{\beta} & =\frac{\sum_{\boldsymbol{x}^{\prime} \in D^{\prime}} F_{\beta}\left(h\left(\boldsymbol{x}^{\prime}\right), \boldsymbol{y}^{\prime}\right)}{\left|D^{\prime}\right|} .
\end{aligned}
$$

The most frequently used F-measure is $F_{1}$.

Other performance measures can also be defined using the contingency matrices (2). This is the case of the Accuracy and Hamming loss.

Definition 4 The Accuracy [26] (or the Jaccard index) is a slight modification of the $F_{1}$ measure defined by

$$
A c\left(h\left(\boldsymbol{x}^{\prime}\right), \boldsymbol{y}^{\prime}\right)=\frac{a}{a+b+c} ; \quad A c=\frac{A c\left(h\left(\boldsymbol{x}^{\prime}\right), \boldsymbol{y}^{\prime}\right)}{\left|D^{\prime}\right|} \text {. }
$$

Definition 5 The Hamming loss measures the proportion of misclassifications,

$$
\begin{aligned}
H l\left(h\left(\boldsymbol{x}^{\prime}\right), \boldsymbol{y}^{\prime}\right) & =\frac{b+c}{a+b+c+d} ; \\
H l & =\frac{\sum_{\boldsymbol{x}^{\prime} \in D^{\prime}} H l\left(h\left(\boldsymbol{x}^{\prime}\right), \boldsymbol{y}^{\prime}\right)}{\left|D^{\prime}\right|} .
\end{aligned}
$$

Throughout the paper we use the term loss function to mean a measure for which lower values are preferable, as happens with the $0 / 1$ and the Hamming losses. On the other hand, we reserve the term score function for $F_{\beta}$ where higher values mean better performance.

\section{Structural Approach for Multilabel Classification}

In this section we follow $[14,13]$ to learn a hypothesis $h$ from an input space $\mathcal{X}$ to an output space $\mathcal{Y}$ endowed with some kind of structure. For instance, the outputs may be parsing trees, or a total ordering in web search, or an alignment between two amino acid sequences in protein threading. But $\mathcal{Y}$ may be as simple as a set of classes; thus multiclass and multilabel classification are included in this framework.

The core idea to learn structured predictions is to induce a discriminant function

$f: \mathcal{X} \times \mathcal{Y} \longrightarrow \mathbb{R}$

that gives rise to a prediction hypothesis

$$
\begin{aligned}
h: \mathcal{X} & \longrightarrow \mathcal{Y} \\
& \boldsymbol{x} \longrightarrow h(\boldsymbol{x})=\underset{\boldsymbol{y} \in \mathcal{Y}}{\operatorname{argmax}} f(\boldsymbol{x}, \boldsymbol{y}) .
\end{aligned}
$$

The intended meaning is to predict for $\boldsymbol{x}$ the output $\boldsymbol{y}$ with the highest $f$-value. Thus, the discriminant function becomes, de facto, a general matching or similarity function.

A straightforward approach to represent a similarity function is by means of a bilinear map. In fact, these maps are a generalization of inner products extended to a couple of different spaces. Thus, if we assume that both the input and the output are vector spaces, a discriminant function will be a bilinear function

$f_{b l}: \mathcal{X} \times \mathcal{Y} \longrightarrow \mathbb{R}$

But bilinear functions from a product space are in oneto-one correspondence with linear functions from the tensor product space. Thus, discriminant functions can be thought as linear maps

$f_{l i}: \mathcal{X} \otimes \mathcal{Y} \longrightarrow \mathbb{R}$ 
The identification of both ways to represent discriminant functions is given by

$f(\boldsymbol{x}, \boldsymbol{y})=f_{b l}(\boldsymbol{x}, \boldsymbol{y})=f_{l i}(\boldsymbol{x} \otimes \boldsymbol{y})=\langle\boldsymbol{w}, \boldsymbol{x} \otimes \boldsymbol{y}\rangle$,

for some director vector $\boldsymbol{w} \in \mathcal{X} \otimes \mathcal{Y}$.

In the literature of structured output learning, discriminant functions are presented as linear maps from a Hilbert space to $\mathbb{R}$

$f(\boldsymbol{x}, \boldsymbol{y})=\langle\boldsymbol{w}, \Psi(\boldsymbol{x}, \boldsymbol{y})\rangle$.

In this context $\Psi(\boldsymbol{x}, \boldsymbol{y})$ is a feature vector in the joint feature space relating inputs and outputs. In our study we have applied the tensor product as a straightforward definition of such joint feature space,

$\Psi(\boldsymbol{x}, \boldsymbol{y})=\boldsymbol{x} \otimes \boldsymbol{y}$

To illustrate this formal framework, let us assume that $\boldsymbol{x} \in \mathcal{X}=\mathbb{R}^{p}$ and $\boldsymbol{y} \in \mathcal{Y}=\mathbb{R}^{L}$. The Kronecker product of these vectors is a representation of the tensor product by another Euclidean vector:

$\boldsymbol{x} \otimes \boldsymbol{y}=(\boldsymbol{x} \cdot \boldsymbol{y}[1], \ldots, \boldsymbol{x} \cdot \boldsymbol{y}[L])$,

SO

$\boldsymbol{x} \otimes \boldsymbol{y} \in\left(\mathbb{R}^{p}\right)^{L}=\mathbb{R}^{(p \times L)} \cong \mathbb{R}^{p} \otimes \mathbb{R}^{L}=\mathcal{H}$.

To interpret a discriminant function in this context, let us assume that $\boldsymbol{w} \in \mathcal{H}$ is given by

$\boldsymbol{w}=\left(\boldsymbol{w}_{1}, \ldots, \boldsymbol{w}_{L}\right), \quad \boldsymbol{w}_{l} \in \mathbb{R}^{p}, l \in \mathscr{L}$.

Then we have that

$f(\boldsymbol{x}, \boldsymbol{y})=\langle\boldsymbol{w},(\boldsymbol{x} \otimes \boldsymbol{y})\rangle=\sum_{l=1}^{L}\left\langle\boldsymbol{w}_{l}, \boldsymbol{x}\right\rangle \cdot \boldsymbol{y}[l]$

Sometimes it is useful to write $f$ in terms of the image of some tensor products. For this purpose, let $\left\{\boldsymbol{e}_{l}: l=1, \ldots, L\right\}$ be the canonical basis of $\mathcal{Y}=\mathbb{R}^{L}$, then the prediction hypothesis (3) is given by

$$
\begin{aligned}
h(\boldsymbol{x}) & =\underset{\boldsymbol{y} \in \mathcal{Y}}{\operatorname{argmax}} f(\boldsymbol{x}, \boldsymbol{y}) \\
& =\underset{\boldsymbol{y} \in \mathcal{Y}}{\operatorname{argmax}} f_{b l}\left(\boldsymbol{x}, \sum_{l=1}^{L} \boldsymbol{e}_{l} \boldsymbol{y}[l]\right) \\
& =\underset{\boldsymbol{y} \in \mathcal{Y}}{\operatorname{argmax}} \sum_{l=1}^{L}\left\langle\boldsymbol{w}, \boldsymbol{x} \otimes \boldsymbol{e}_{l}\right\rangle \cdot \boldsymbol{y}[l] \\
& =\left\{l \in \mathscr{L}:\left\langle\boldsymbol{w}, \boldsymbol{x} \otimes \boldsymbol{e}_{l}\right\rangle>0\right\} \\
& =\left\{l \in \mathscr{L}:\left\langle\boldsymbol{w}_{l}, \boldsymbol{x}\right\rangle>0\right\} .
\end{aligned}
$$

Let us remark that there is an interesting and alternative way to present a discriminant function. If vectors $\boldsymbol{x}$ and $\boldsymbol{y}$ are column vectors, and $\boldsymbol{W}$ is the matrix whose columns are $\boldsymbol{w}_{1}, \ldots, \boldsymbol{w}_{L}(4)$, then

$f(\boldsymbol{x}, \boldsymbol{y})=\sum_{l=1}^{L}\left\langle\boldsymbol{w}_{l}, \boldsymbol{x}\right\rangle \cdot \boldsymbol{y}[l]=\boldsymbol{x}^{T} \boldsymbol{W} \boldsymbol{y}$

This is the typical representation of bilinear functions. Finally, notice that when for each output we have that

$\sum_{l \in \mathscr{L}} \boldsymbol{y}[l]=1$

then the multilabel task is just a multiclass classification task. As was pointed out by Joachims et al. [14], the approach presented here is then identical to the multiclass approach of Crammer and Singer [2].

In the preceding derivation, we lost the intercept terms frequently used in linear hypotheses. Thus, we add a new constant feature for all vectors $\boldsymbol{x}$. This trick recovers the intercept terms; therefore, in practice,

$\mathcal{X} \subset \mathbb{R}^{p+1}$

\section{Learning Discriminant Functions}

A general approach to learn the parameter $\boldsymbol{w}$ is to use a regularized risk minimization method [29]. The aim is to search for a model that minimizes the average prediction loss in the training set plus a quadratic regularizer that penalizes complex solutions. There are different optimization problems that formalize the search for an optimal $\boldsymbol{w}$. We shall use the 1-slack structural SVM with margin-rescaling [14]:

$$
\begin{aligned}
& \min _{\boldsymbol{w}, \xi \geq 0} \frac{1}{2}\langle\boldsymbol{w}, \boldsymbol{w}\rangle+C \xi \\
& \text { s.t. } \frac{1}{n}\left\langle\boldsymbol{w}, \sum_{i=1}^{n}\left[\Psi\left(\boldsymbol{x}_{i}, \boldsymbol{y}_{i}\right)-\Psi\left(\boldsymbol{x}_{i}, \overline{\boldsymbol{y}}_{i}\right)\right]\right\rangle \geq \frac{1}{n} \sum_{i=1}^{n} \Delta\left(\boldsymbol{y}_{i}, \overline{\boldsymbol{y}}_{i}\right)-\xi \\
& \forall\left(\overline{\boldsymbol{y}}_{1}, \ldots \overline{\boldsymbol{y}}_{n}\right) \in \mathcal{Y}^{n} .
\end{aligned}
$$

This problem can be solved in time linear in $n$ using SVM $^{\text {struct }}[14]$.

Let us emphasize that in this approach to multilabel classification, the loss function $\Delta$ refers to examplebased errors (see Section 2.1). In fact, the average loss is bounded by the parameter $\xi$ that must be minimized according to (6). To see this, let us observe that the constraints of (6) ensure that for every collection of $n$ subsets of the label set, $\forall\left(\overline{\boldsymbol{y}}_{1}, \ldots \overline{\boldsymbol{y}}_{n}\right) \in \mathcal{Y}^{n}$, we have that

$\xi \geq \frac{1}{n} \sum_{i=1}^{n} \Delta\left(\boldsymbol{y}_{i}, \overline{\boldsymbol{y}}_{i}\right)-\frac{1}{n}\left\langle\boldsymbol{w}, \sum_{i=1}^{n}\left[\Psi\left(\boldsymbol{x}_{i}, \boldsymbol{y}_{i}\right)-\Psi\left(\boldsymbol{x}_{i}, \overline{\boldsymbol{y}}_{i}\right)\right]\right\rangle$. 
On the other hand, given the definition of the prediction hypothesis $(3)$, for $h\left(\boldsymbol{x}_{i}\right)=\overline{\boldsymbol{y}}_{i}$ we obtain that

$0 \geq \frac{1}{n}\left\langle\boldsymbol{w}, \sum_{i=1}^{n}\left[\Psi\left(\boldsymbol{x}_{i}, \boldsymbol{y}_{i}\right)-\Psi\left(\boldsymbol{x}_{i}, h\left(\boldsymbol{x}_{i}\right)\right)\right]\right\rangle$.

Thus

$\xi \geq \frac{1}{n} \sum_{i=1}^{n} \Delta\left(\boldsymbol{y}_{i}, h\left(\boldsymbol{x}_{i}\right)\right)$

\subsection{Optimization Algorithm}

As was mentioned above, there is an efficient algorithm to find the optimum parameters in (6) for a given $\Delta$ loss function. This job is done by the cutting plane algorithm (Alg. 1) of [14]. The core point is the argmax step (line 6 in Alg. 1) where the algorithm looks for the most violated constraint. This step is specific for each loss function and should be computationally efficient.

For each element of the multilabel task $D(1)$ the purpose is to find the output with the highest value of the sum of the loss plus the discriminant function. To avoid unnecessary subindexes, let $(\boldsymbol{x}, \boldsymbol{y})$ be an arbitrary training pair of $D$. Then, for $\boldsymbol{w} \in \mathcal{H}$, the aim is to solve

$$
\begin{aligned}
& \underset{\hat{\boldsymbol{y}} \in \mathcal{Y}}{\operatorname{argmax}}\{\Delta(\boldsymbol{y}, \hat{\boldsymbol{y}})+\langle\boldsymbol{w}, \Psi(\boldsymbol{x}, \hat{\boldsymbol{y}})\rangle\}= \\
& \underset{\hat{\boldsymbol{y}} \in \mathcal{Y}}{\operatorname{argmax}}\left\{\Delta(\boldsymbol{y}, \hat{\boldsymbol{y}})+\sum_{l=1}^{L}\left\langle\boldsymbol{w}, \boldsymbol{x} \otimes \boldsymbol{e}_{l}\right\rangle \cdot \hat{\boldsymbol{y}}[l]\right\} .
\end{aligned}
$$

Once this step is performed for all training examples, the most violated constraint corresponds to the output formed by the joint of the outputs returned by (7) for each element in $D$.

Therefore, we are able to build specific algorithms to optimize example-based loss functions whenever we can devise algorithms to compute the argmax of (7). In the following subsections we present algorithms for different loss functions.

\subsection{Subset 0/1 Loss}

In this case, the loss is 1 if $\boldsymbol{y} \neq \hat{\boldsymbol{y}}$, otherwise it is 0 . Thus, (7) becomes

$\underset{\hat{\boldsymbol{y}} \in \mathcal{Y}}{\operatorname{argmax}}\left\{\llbracket \boldsymbol{y} \neq \hat{\boldsymbol{y}} \rrbracket+\sum_{l=1}^{L}\left\langle\boldsymbol{w}, \boldsymbol{x} \otimes \boldsymbol{e}_{l}\right\rangle \cdot \hat{\boldsymbol{y}}[l]\right\}$.

To maximize the sum, define $\tilde{\boldsymbol{y}}$ such that

$\forall l \in\{1, \ldots, n\}, \quad \tilde{\boldsymbol{y}}[l]=\llbracket\left\langle\boldsymbol{w}, \boldsymbol{x} \otimes \boldsymbol{e}_{l}\right\rangle>0 \rrbracket$.

If $\boldsymbol{y} \neq \tilde{\boldsymbol{y}}$, then the loss is 1 , the maximum possible value. Otherwise the loss is 0 ; in this case, let $\tilde{\boldsymbol{y}}^{\prime}$ be $\tilde{\boldsymbol{y}}$

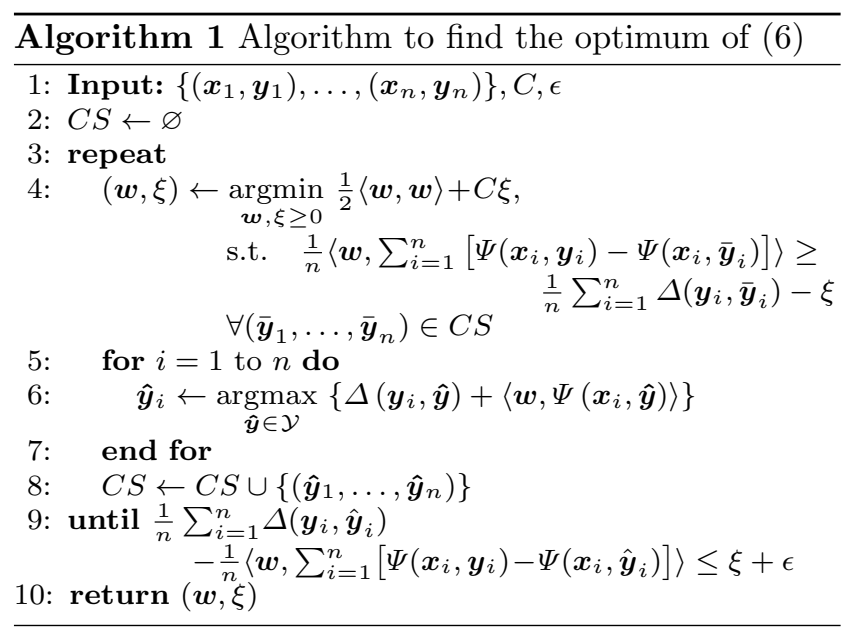

swapping the component with the lowest influence in the sum. In symbols, swap the component with index

$\tilde{l}=\underset{l \in\{1, \ldots, n\}}{\operatorname{argmin}}\left|\left\langle\boldsymbol{w}, \boldsymbol{x} \otimes \boldsymbol{e}_{l}\right\rangle\right|$.

In this situation, for $\tilde{\boldsymbol{y}}^{\prime}$ against $\tilde{\boldsymbol{y}}$, we obtain an increase of 1 (due to the loss) in the expression to be maximized, but a decrease of $\left|\left\langle\boldsymbol{w}, \boldsymbol{x} \otimes \boldsymbol{e}_{\tilde{l}}\right\rangle\right|$. Thus, we select the most convenient vector as the result for the argmax problem (8).

\subsection{Hamming Loss}

The argmax problem can now be written as follows.

$\underset{\hat{\boldsymbol{y}} \in \mathcal{Y}}{\operatorname{argmax}}\left\{\sum_{l=1}^{L} \frac{\llbracket \boldsymbol{y}[l] \neq \hat{\boldsymbol{y}}[l] \rrbracket}{L}+\sum_{l=1}^{L}\left\langle\boldsymbol{w}, \boldsymbol{x} \otimes \boldsymbol{e}_{l}\right\rangle \cdot \hat{\boldsymbol{y}}[l]\right\}=$
$\underset{\hat{\boldsymbol{y}} \in \mathcal{Y}}{\operatorname{argmax}}\left\{\sum_{l=1}^{L}\left(\frac{\llbracket \boldsymbol{y}[l] \neq \hat{\boldsymbol{y}}[l] \rrbracket}{L}+\left\langle\boldsymbol{w}, \boldsymbol{x} \otimes \boldsymbol{e}_{l}\right\rangle \cdot \hat{\boldsymbol{y}}[l]\right)\right\}=$
$\underset{\hat{\boldsymbol{y}}[l] \in\{0,1\}}{\operatorname{argmax}}\left\{\frac{\llbracket \boldsymbol{y}[l] \neq \hat{\boldsymbol{y}}[l] \rrbracket}{L}+\left\langle\boldsymbol{w}, \boldsymbol{x} \otimes \boldsymbol{e}_{l}\right\rangle \cdot \hat{\boldsymbol{y}}[l]\right\}: l=1, \ldots, L$.

That is to say, the argmax problem for Hamming loss can be solved point-wise. There is no multilabel effect here. The predictions about one label do not affect other labels in any sense. This was already acknowledged in [3].

\subsection{Example-Based on Contingency Matrices}

There is a number of loss functions that can be computed from contingency matrices including $F_{1}$, Accuracy, Precision, and Recall, see Section 2.1. In these cases, the argmax step of Algorithm 1 can be computed 


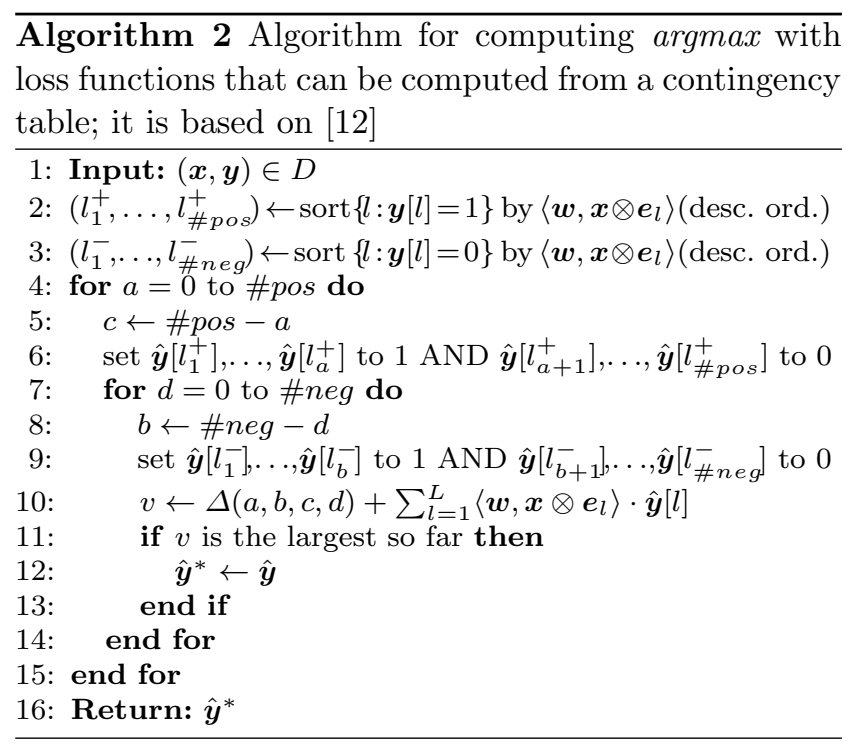

using the idea of Joachims in [12] (Algorithm 2, and Lemma 1).

The core observation is that the computation of the argmax can be done iterating over all possible contingency tables. Thus, for each contingency table of values $(a, b, c, d)$, it is sufficient to compute the output $\hat{\boldsymbol{y}}$ with the highest value of the discriminant function. Therefore,

$$
\begin{aligned}
& \underset{\hat{\boldsymbol{y}} \in \mathcal{Y}}{\operatorname{argmax}}\left\{\Delta(\boldsymbol{y}, \hat{\boldsymbol{y}})+\sum_{l=1}^{L}\left\langle\boldsymbol{w}, \boldsymbol{x} \otimes \boldsymbol{e}_{l}\right\rangle \cdot \hat{\boldsymbol{y}}[l]\right\}= \\
& \underset{\hat{\boldsymbol{y}} \in \mathcal{Y}}{\operatorname{argmax}}\left\{\max _{(a, b, c, d)}\{\Delta(a, b, c, d)+\right. \\
& \left.\left.\max _{\hat{\boldsymbol{y}} \in \mathcal{Y}_{(a, b, c, d)}} \sum_{l=1}^{L}\left\langle\boldsymbol{w}, \boldsymbol{x} \otimes \boldsymbol{e}_{l}\right\rangle \cdot \hat{\boldsymbol{y}}[l]\right\}\right\} .
\end{aligned}
$$

Hence, we need to compute the output $\hat{\boldsymbol{y}}$ with the highest value for those that produce a given contingency matrix:

$\underset{\hat{\boldsymbol{y}} \in \mathcal{Y}_{(a, b, c, d)}}{\operatorname{argmax}} \sum_{l=1}^{L}\left\langle\boldsymbol{w}, \boldsymbol{x} \otimes \boldsymbol{e}_{l}\right\rangle \cdot \hat{\boldsymbol{y}}[l]$.

The maximum value is achieved when the true positive hits $(a)$ are obtained with those labels $l$ with the highest value in

$\left\langle\boldsymbol{w}, \boldsymbol{x} \otimes \boldsymbol{e}_{l}\right\rangle \cdot \hat{\boldsymbol{y}}[l]$

and the true negatives correspond to labels with the lowest value in that amount. Thus, Algorithm 2 computes the argmax step for optimizing example-based loss functions that can be computed using a contingency table.

The complexity of this algorithm for solving the argmax problem is related to $L$, the number of labels.
In the worst case it is $O\left(L^{2}\right)$. Thus, the multilabel classifier can be computed in $O\left(n \cdot L^{2}\right)$ using Algorithms 1 and 2 .

\section{Related Work}

There are many ML learning algorithms, some early approaches have been proposed by Schapire and Singer [24] and by Elisseeff and Weston [8]. There have been proposals from different points of view: nearest neighbors [32], logistic regression [1], Bayesian methods [3, 31 ], using some kind of stacking or chaining iterations $[19,23,18]$, extending multiclass classifications [28]. A review of ML learning methods can be seen in [27].

However this paper is more closely related to those approaches that explicitly aim to optimize a target loss function in the ML arena. There are also some theoretical studies, like $[3,9,5,6]$. Other alternative approaches consist of searching for an optimal thresholding after ranking the set of labels to be assigned to an instance $[8,22]$.

The approach of structured output using tensor products has been used before. For instance, in [11], tensor products were used to derive a learner for optimizing loss functions that decompose over the individual labels. This yields to deal with macro-averages and Hamming loss, that can be optimized by a Binary Relevance method.

Petterson and Caetano present, in [20], a similar approach but limited to optimize macro-averaged loss functions and in [21] also propose to optimize examplebased F1 using the correlations of some sets of labels. These approaches are compared, in [4], with a consistent Bayesian algorithm that estimates all parameters required for a Bayes-optimal prediction via a set of multinomial regression models. The difference between our proposal and [4], is that our method intends to maximize example-based F1 measure during learning (following a structured loss minimization approach) whereas the method presented in [4] makes F-measure maximizing predictions from probabilistic models during an inference step (following a plug-in rule approach). Thus, both methods are quite different and our proposal is more related to [21]).

Notice that the objective of this paper is not to compare a particular couple of learners but to study the relations between loss functions and optimization aims; thus we need a common framework.

The use of structured outputs in this paper follows the works by Joachims and co-workers [25, 13, 14]. The optimization of multivariate loss functions was also adapted from the paper [12]. 
Other structured output approaches can be found in [15] and in [10] (in this case using Conditional Random Fields). In these cases, an important issue to be addressed is the inference problem, that is, to determine the most likely output for a given input.

\section{Experimental Results}

In this section we present a number of experimental results devised to show the performance of the structural optimization approaches discussed in this paper. The purpose is to show the differences in performance when the aim is to optimize one specific measure while the assessment is done using either the same or another measure. For this purpose we implemented three learners to optimize the Hamming loss, $F_{1}$ score, and $0 / 1$ loss respectively. We shall refer to these systems by $\operatorname{Struct}(H l), \operatorname{Struct}\left(F_{1}\right)$, and $\operatorname{Struct}(01)$ respectively.

The implementation was done using the svm-structmatlab of [30], a MATLAB wrapper of SVM ${ }^{\text {struct }}[25$, 14]. In this framework, we codified the argmax functions detailed in sections 4.2, 4.3, and 4.4 (Algorithm 2). In the experiments reported in this section, $\mathrm{SVM}^{\text {struct }}$ was used with parameters $\epsilon=0.1$, margin rescaling, and 1slack formulation with constraint cache.

\subsection{Datasets}

The experiments were done using synthetic datasets, we preferred them to 'real' datasets. The reason is that we want to provide a strong support using data with a variety of characteristics since we are trying to show the influence of those characteristics on the performance of the learners.

We used a synthetic dataset generator to build 48 datasets: each one composed by a training, a validation and a testing set. In all cases the input space was $\mathbb{R}^{p+1}$ with $p=25$, see (5). The size $L$ of the set of labels varied in $\{10,25,50\}$; we generated 16 datasets for each number of labels. The target cardinalities (average number of labels per example) ranged from 2 to 4 . In Table 1 it is shown maximum, minimum, average and standard deviation of cardinality and density in these datasets grouped by the number of labels. The collection of datasets used in the experiments is publicly available in our website with an implementation and a detailed description of the generator ${ }^{1}$.

The generator draws a set of 400 points in $[0,1]^{p}$ that constitute the inputs of the training set. Then, using a genetic algorithm, it searches for $L$ hyperplanes

\footnotetext{
1 http://www.aic.uniovi.es/ml_generator/
}

such that the set of labels so obtained fits as much as possible to the target characteristics. Those hyperplanes are then used to generate the validation and testing sets with 400 and 600 examples respectively.

In addition to these 48 datasets we used noisy versions of them. The noise was added to training and validation sets using two procedures: Bernoulli and Swap. The first procedure uses a Bernoulli distribution with probability $p r$ to change the values (0 and 1$)$ of the matrix of labels. We used

$p r \in\{0.01,0.03,0.05,0.07,0.09\}$.

On the other hand, the Swap procedure adds noise interchanging a couple of $(0,1)$ values in the same example with probability $p r$; notice that this method preserves the cardinality of the datasets. In this case,

$p r \in\{0.05,0.10,0.20,0.30,0.40\}$.

Thus, we have 528 datasets.

In general, the role of noise is to make harder a learning task. Of course, the effect of these two kinds of noise is not the same: it should depend on the value of $p r$ (we can somehow consider it captures the intensity of the effect), but also on the type of noise and even on the loss function used to measure the performance.

\subsection{Results and Discussion}

For each of the 528 datasets we computed the score in the test set after adjusting the $C$ parameter (6) using train and validation sets. We searched for $C$ values in $\left\{10^{i}: i=4,5,6\right\}$ for noise free datasets and $\left\{10^{i}: i=\right.$ $2,3,4,5\}$ for noisy ones.

In Table 2 we reported the average values of the performance of the learners measured with Hamming loss, $0 / 1$ loss, and the example-based $F_{1}$ loss in a traintest experiment; that is, $1-F_{1}$. All values are expressed as percentages for ease of reading. The averages are computed for each measure function, number of labels and type of noise.

Struct(01) reaches the best scores for all performance measures in noise free datasets; these can be considered easier learning tasks. Thus, when a hypothesis correctly predicts the whole set of labels, it achieves the best possible results independently of the measure used to assess the performance. Even with low noise this is the best learner, although the differences are very small in all cases.

However, as the degree of noise increases, the performance of Struct(01) drops substantially. In noisy data it is a good option to optimize Hamming loss or the $F_{1}$ 
Table 1 Cardinality and density statistics of the 48 free-noise datasets. Datasets with Bernoulli and swap noise present similar figures

\begin{tabular}{lcc}
\hline & Cardinality & Density \\
\hline 50 Labels & & \\
\hline max & 4.3 & $9 \%$ \\
min & 2.5 & $5 \%$ \\
mean & 3.3 & $7 \%$ \\
std dev & 0.5 & $1 \%$ \\
\hline 25 Labels & & \\
\hline max & 4.3 & $17 \%$ \\
min & 2.4 & $10 \%$ \\
mean & 3.1 & $13 \%$ \\
std dev & 0.6 & $2 \%$ \\
\hline 10 Labels & & \\
\hline max & 4.0 & $40 \%$ \\
min & 1.8 & $18 \%$ \\
mean & 2.9 & $29 \%$ \\
std dev & 0.7 & $7 \%$ \\
\hline
\end{tabular}

score, despite the performance measure used to assess the classifier.

To see at a glance the whole bunch of results, we show Figures 1 and 2. Here we represent the average scores for increasing levels of noise obtained by the learners $\operatorname{Struct}(H l), \operatorname{Struct}\left(F_{1}\right)$, and $\operatorname{Struct}(01)$.

In these figures we see that $\operatorname{Struct}(H l)$ is a quite competitive learner. As expected, it is the best when the purpose is to win in Hamming loss, but the scores obtained with the other performance measures are very good too. In fact, $\operatorname{Struct}(\mathrm{Hl})$ outperforms the other options in datasets with some noise when the performance measure is $0 / 1$ loss. In $F_{1}$, $\operatorname{Struct}(H l)$ outperforms the scores of $\operatorname{Struct}\left(F_{1}\right)$ when the learning task is easier, with a small proportion of noisy examples. Only when the learning task has high levels of noise, there is a room for improvement: $\operatorname{Struct}\left(F_{1}\right)$ is better in these cases. Nevertheless, there are no big differences and a tradeoff between performance and algorithmic complexity may be favorable to $\operatorname{Struct}(H l)$.

The competitive performance showed by $\operatorname{Struct}(H l)$ on example-based measures is in line with the results of BR reported in the experimental study [17]. Let us recall that $\operatorname{Struct}(\mathrm{Hl})$ is conceptually equivalent to a BR (Section 2.1) with the only difference that $\operatorname{Struct}(H l)$ performs a combined regularization for all models defined in (4).

In general, since the optimization of the Hamming loss produces competitive scores, a simple Binary Relevance classifier is useful to solve ML tasks (this conclusion was also reached in [16]). This is not the case when the aim is to minimize the subset $0 / 1$ loss with- out noise. In that case the learner optimizing 0/1 loss performs better.

The Appendix at the end of the paper contains a detailed graphical description of the winning regions of each learner in the whole collection of datasets used in the experiments.

\section{Conclusions}

There is a tradeoff between performance and computational complexity in classification tasks. When the goal is to optimize a loss function defined over the whole classification of a set, as in the F-measures, the learner has to deal with contingency tables. If the aim is to predict a set of labels instead of a single class, the algorithms become more complex.

We have presented some contributions to guide the search of a tradeoff in ML classification. On the one hand, we implemented, using a common framework, three learners that explicitly optimize three of the most popular performance measures of ML classifiers, respectively: subset 0/1 loss, Hamming loss, and the examplebased F-measure. For this purpose we used Structured output Support Vector Machines (SSVM) and extended to ML the method presented by Joachims in [12] to optimize multivariate performance measures. Then, to compare these learners in a wide variety of situations, we used a collection of 528 synthetic ML datasets. Here we included different levels of noise and number of labels.

The results of the comparison are detailed in Section 6 and in the Appendix at the end of the paper. 
Table 2 Average train-test results in different noise settings in 528 datasets. The learners used aim to optimize Hamming loss, $F_{1}$ score, and $0 / 1$ loss. The performance measures are $0 / 1$ loss $(0 / 1)$, Hamming loss (Hl) and $1-F_{1}$. Noise was added using Bernoulli and Swap (Section 6.1) procedures

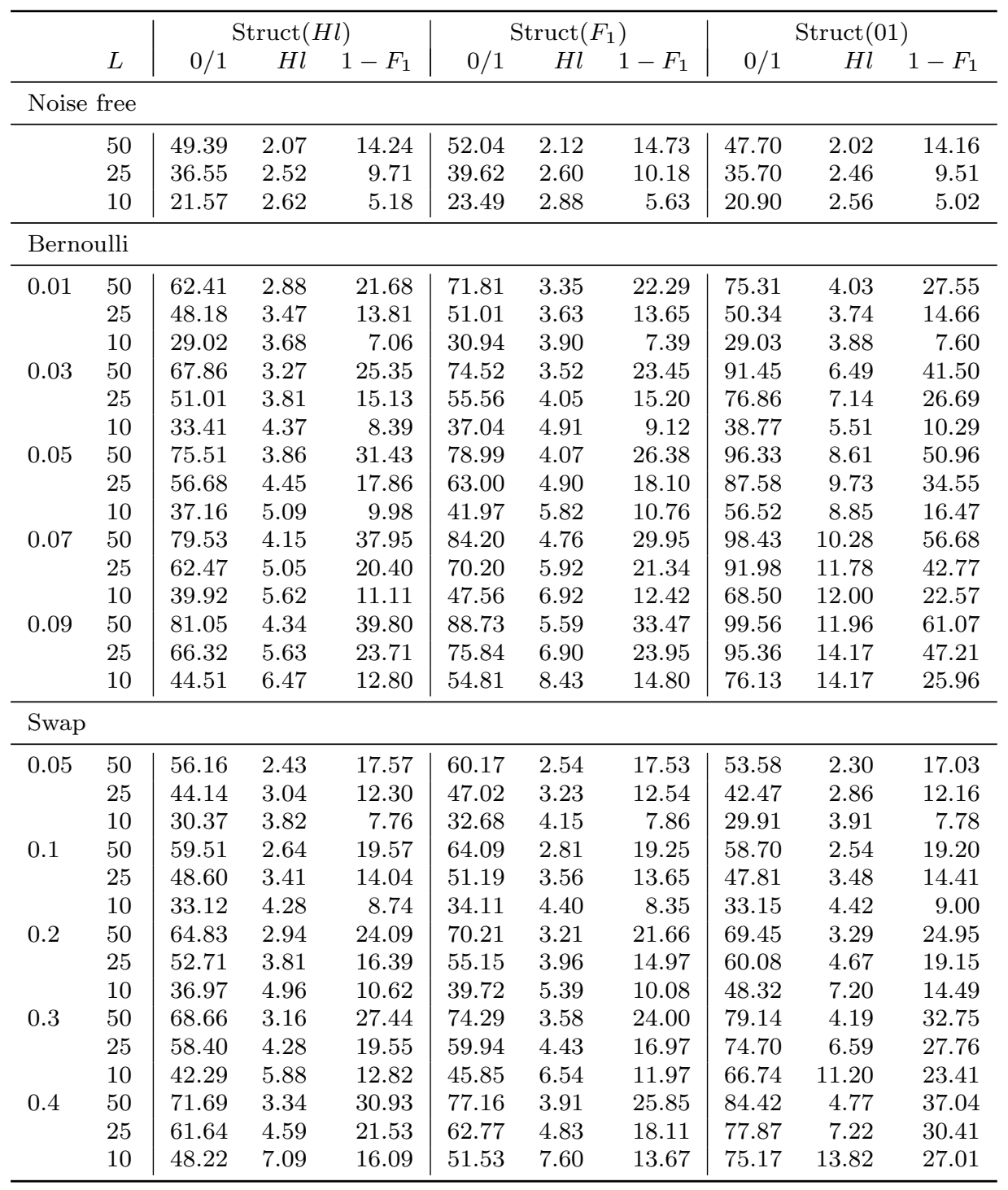

But the overall conclusions can be summarized in a few words.

In most cases, the optimization of the Hamming loss produces the best or competitive scores. Notice that this is very important in a practical sense since a simple Binary Relevance classifier can be used for this purpose. Any binary classifier can be utilized to learn the relevancy of each label separately. Thus, ML tasks can be tackled with a scalable and effective method most of the times.

The limits of this general rule are when the aim is to minimize the subset $0 / 1$ loss in noise-free datasets. In these cases, the specialized learner Struct(01) achieves the best results, although the differences are very small. Bigger differences are appreciated when learning tasks have high levels of noise and the purpose is to improve the performance in F-measure. In this case the learner that explicitly optimizes this loss outperforms the others.

Acknowledgements The research reported here is supported in part under grant TIN2011-23558 from the MINECO (Ministerio de Economía y Competitividad, Spain), partially supported with FEDER funds. We would also like to acknowledge all those people who generously shared the datasets and software used in this paper.

\section{References}

1. Cheng, W., Hüllermeier, E.: Combining Instance-Based Learning and Logistic Regression for Multilabel Classifi- 

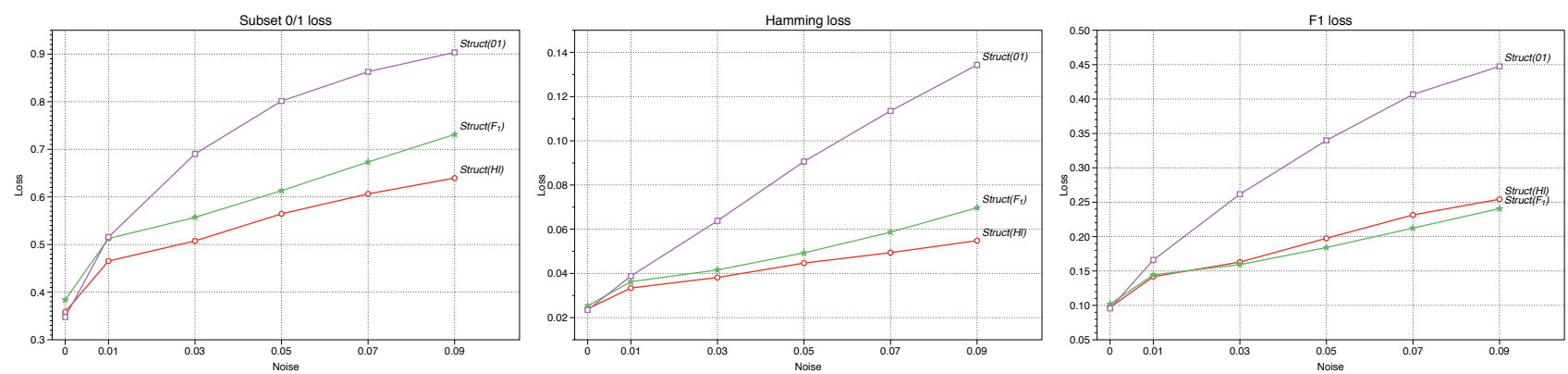

Fig. 1 Average scores in datasets with different Bernoulli noise levels (horizontal axis), see Section 6.1. In the leftmost picture we represent $0 / 1$ loss, in the central the Hamming loss, and $1-F_{1}$ in the rightmost picture. Since all the scores represent losses, the lower is the better
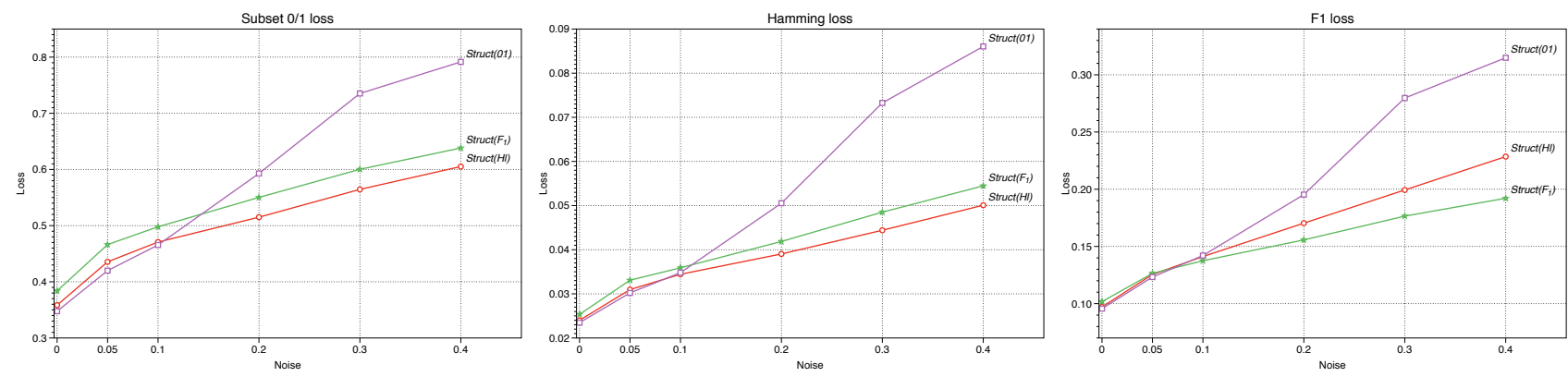

Fig. 2 Average scores in datasets with different Swap noise levels (horizontal axis), see Section 6.1. In the leftmost picture we represent $0 / 1$ loss, in the central the Hamming loss, and $1-F_{1}$ in the rightmost picture. Since all the scores represent losses, the lower is the better

cation. Machine Learning 76(2), 211-225 (2009)

2. Crammer, K., Singer, Y.: On the algorithmic implementation of multiclass kernel-based vector machines. Journal of Machine Learning Research 2, 265-292 (2002)

3. Dembczyński, K., Cheng, W., Hüllermeier, E.: Bayes optimal multilabel classification via probabilistic classifier chains. In: Proceedings of the International Conference on Machine Learning (ICML) (2010)

4. Dembczyński, K., Kotłowski, W., Jachnik, A., Waegeman, W., Hüllermeier, E.: Optimizing the f-measure in multi-label classification: Plug-in rule approach versus structured loss minimization. ICML (2013)

5. Dembczyński, K., Waegeman, W., Cheng, W., Hüllermeier, E.: An Exact Algorithm for F-Measure Maximization. In: Proceedings of the Neural Information Processing Systems (NIPS) (2011)

6. Dembczyński, K., Waegeman, W., Cheng, W., Hüllermeier, E.: On label dependence and loss minimization in multi-label classification. Machine Learning pp. $1-41(2012)$

7. Díez, J., del Coz, J.J., Luaces, O., Bahamonde, A.: Tensor products to optimize label-based loss measures in multilabel classifications. Tech. rep., Centro de Inteligencia Artificial. Universidad de Oviedo at Gijón (2012)

8. Elisseeff, A., Weston, J.: A kernel method for multilabelled classification. In: Proceedings of the Annual Conference on Neural Information Processing Systems (NIPS), pp. 681-687. MIT Press (2001)

9. Gao, W., Zhou, Z.H.: On the consistency of multi-label learning. Journal of Machine Learning Research - Proceedings Track (COLT) 19, 341-358 (2011)

10. Ghamrawi, N., McCallum, A.: Collective multi-label classification. In: Proceedings of the 14th ACM International
Conference on Information and Knowledge Management, pp. 195-200. ACM (2005)

11. Hariharan, B., Vishwanathan, S., Varma, M.: Efficient max-margin multi-label classification with applications to zero-shot learning. Machine learning $\mathbf{8 8}(1-2), 127-155$ (2012)

12. Joachims, T.: A support vector method for multivariate performance measures. In: Proceedings of the International Conference on Machine Learning (ICML) (2005)

13. Joachims, T.: Training linear SVMs in linear time. In: Proceedings of the ACM Conference on Knowledge Discovery and Data Mining (KDD). ACM (2006)

14. Joachims, T., Finley, T., Yu, C.: Cutting-plane training of structural svms. Machine Learning 77(1), 27-59 (2009)

15. Lampert, C.H.: Maximum margin multi-label structured prediction. In: Advances in Neural Information Processing Systems, pp. 289-297 (2011)

16. Luaces, O., Díez, J., Barranquero, J., del Coz, J.J., Bahamonde, A.: Binary relevance efficacy for multilabel classification. Progress in Artificial Intelligence 4(1), 303-313 (2012)

17. Madjarov, G., Kocev, D., Gjorgjevikj, D., Džeroski, S.: An extensive experimental comparison of methods for multi-label learning. Pattern Recognition 45(9), 3084 - 3104 (2012). DOI http://dx.doi.org/10.1016/j. patcog.2012.03.004. URL http://www.sciencedirect. com/science/article/pii/S0031320312001203

18. Montañés, E., Quevedo, J., del Coz, J.: Aggregating independent and dependent models to learn multi-label classifiers. In: Proceedings of European Conference on Machine Learning and Knowledge Discovery in Databases (ECML-PKDD), pp. 484-500. Springer (2011) 
19. Montañes, E., Senge, R., Barranquero, J., Ramón Quevedo, J., José del Coz, J., Hüllermeier, E.: Dependent binary relevance models for multi-label classification. Pattern Recognition 47(3), 1494-1508 (2014)

20. Petterson, J., Caetano, T.: Reverse multi-label learning. In: Proceedings of the Annual Conference on Neural Information Processing Systems (NIPS), pp. 1912-1920 (2010)

21. Petterson, J., Caetano, T.S.: Submodular multi-label learning. In: Proceedings of the Annual Conference on Neural Information Processing Systems (NIPS), pp. $1512-1520$ (2011)

22. Quevedo, J.R., Luaces, O., Bahamonde, A.: Multilabel classifiers with a probabilistic thresholding strategy. Pattern Recognition 45(2), 876-883 (2012)

23. Read, J., Pfahringer, B., Holmes, G., Frank, E.: Classifier Chains for Multi-label Classification. In: Proceedings of European Conference on Machine Learning and Knowledge Discovery in Databases (ECML-PKDD), pp. 254-269 (2009)

24. Schapire, R., Singer, Y.: Boostexter: A boosting-based system for text categorization. Machine learning 39(2), 135-168 (2000)

25. Tsochantaridis, I., Joachims, T., Hofmann, T., Altun, Y.: Large margin methods for structured and interdependent output variables. Journal of Machine Learning Research 6(2), 1453 (2006)

26. Tsoumakas, G., Katakis, I.: Multi Label Classification: An Overview. International Journal of Data Warehousing and Mining 3(3), 1-13 (2007)

27. Tsoumakas, G., Katakis, I., Vlahavas, I.: Mining Multilabel Data. In O. Maimon and L. Rokach (Ed.), Data Mining and Knowledge Discovery Handbook, Springer (2010)

28. Tsoumakas, G., Katakis, I., Vlahavas, I.: Random kLabelsets for Multi-Label Classification. IEEE Transactions on Knowledge Discovery and Data Engineering (2010)

29. Vapnik, V.: Statistical Learning Theory. John Wiley, New York, NY (1998)

30. Vedaldi, A.: A MATLAB wrapper of SVM ${ }^{\text {struct }}$. http://www.vlfeat.org/ $\quad$ vedaldi/code/svm-structmatlab.html (2011)

31. Zaragoza, J., Sucar, L., Bielza, C., Larrañaga, P.: Bayesian chain classifiers for multidimensional classification. In: Proceedings of the International Joint Conference on Artificial Intelligence (IJCAI) (2011)

32. Zhang, M.L., Zhou, Z.H.: ML-KNN: A Lazy Learning Approach to Multi-label Learning. Pattern Recognition 40(7), 2038-2048 (2007)

\section{Appendix}

In this section we report the results obtained in the whole collection of datasets. Since we have two different ways to introduce noise in a learning task (see Section 6.1), in order to represent all datasets at once, we define the similarity of noise free and noisy versions for each dataset and loss or score function. For a loss function $\Delta$, the similarity is the complementary of the loss of the noisy release with respect to the noise free output in the test set,

$\operatorname{Sim}(\Delta, \mathbf{Y}, \operatorname{noise}(\mathbf{Y}))=1-\Delta(\mathbf{Y}, \operatorname{noise}(\mathbf{Y}))$

where $\mathbf{Y}$ represents the matrix of actual labels. On the other hand, the similarity does not use the complementary in $F_{1}$,

$\operatorname{Sim}\left(F_{1}, \mathbf{Y}, \operatorname{noise}(\mathbf{Y})\right)=F_{1}(\mathbf{Y}, \operatorname{noise}(\mathbf{Y}))$.

In Figure 3 each dataset is represented in a 2-dimension space. The horizontal axis represents the $F_{1}$ score achieved by $\operatorname{Struct}\left(F_{1}\right)$ in the noise free version of the dataset. The vertical axis represents the similarity of the dataset (measured with $F_{1}$ ). Thus, points near the top of the picture stand for datasets noise free or datasets with low noise. On the other hand, points near the left side represent harder datasets, in the sense that the noise free releases achieves lower $F_{1}$ scores. Finally, the points in this space are labeled by the name of the learner that achieved the best $F_{1}$ score.

Here we observe that $\operatorname{Struct}\left(F_{1}\right)$ outperforms the other learners in terms of $F_{1}$ when tackling harder learning tasks (left bottom corner of Figure 3). In easier tasks, mainly those with 10 or 25 labels, the procedure (Algorithm 2) seems to require more evidences in order to estimate the optimal expected $F_{1}$. In any case, the differences in the easiest learning tasks are small.

To complete the discussion of the results, we made figures analogous to Figure 3 using subset 0/1 loss (Fig. 4), and Hamming loss (Fig. 5). 


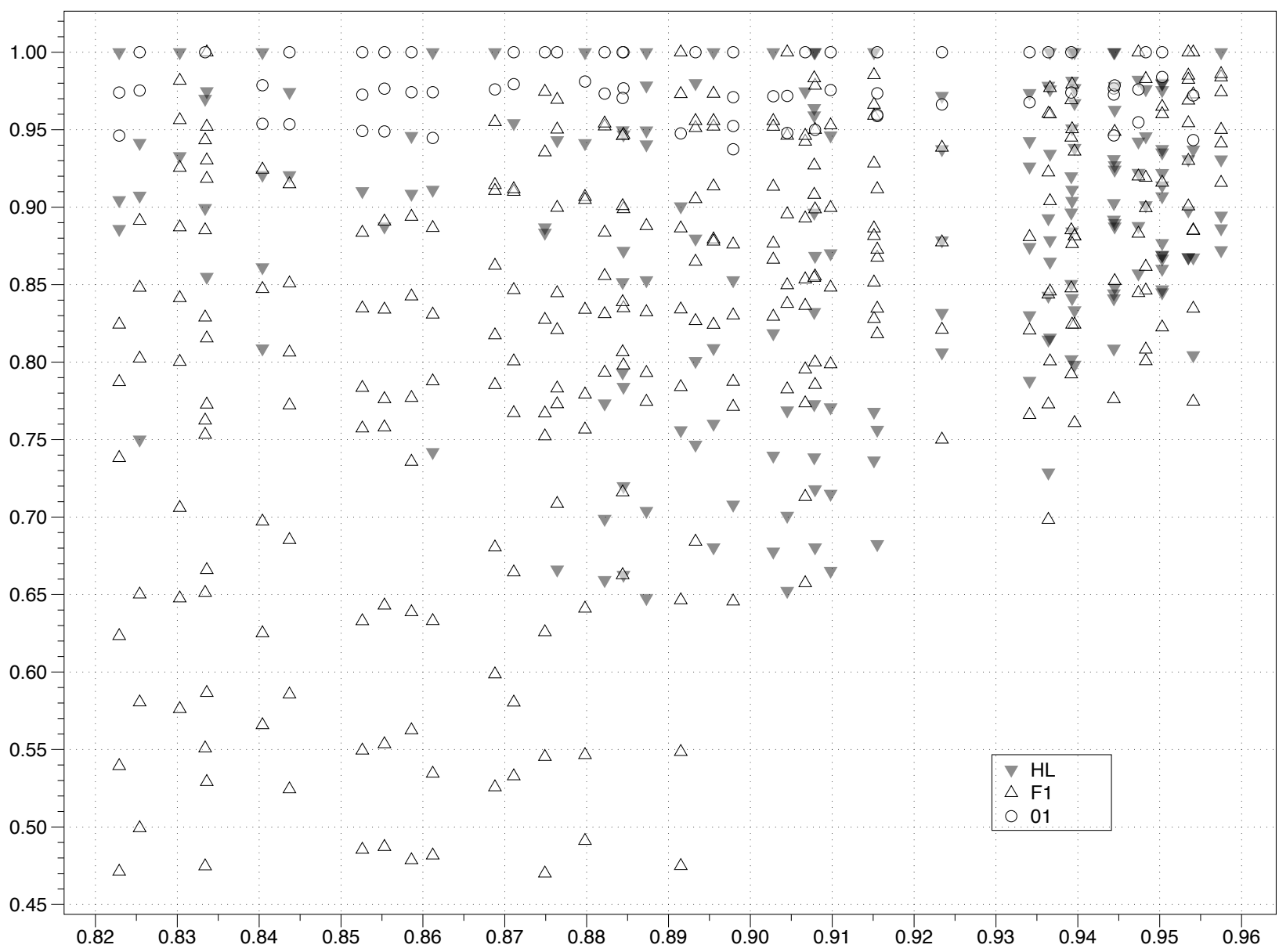

Fig. 3 Learners winning in $F_{1}$ score in the 528 datasets. The horizontal axis represents the $F_{1}$ score of datasets in the noise free releases. Thus points at the right hand side stand for easier datasets, typically those with less number of labels. The vertical axis represent the similarity of label sets, using $F_{1}$ similarity (10), with the noise free version. The higher the points in the figure, the lower the noise in the datasets 


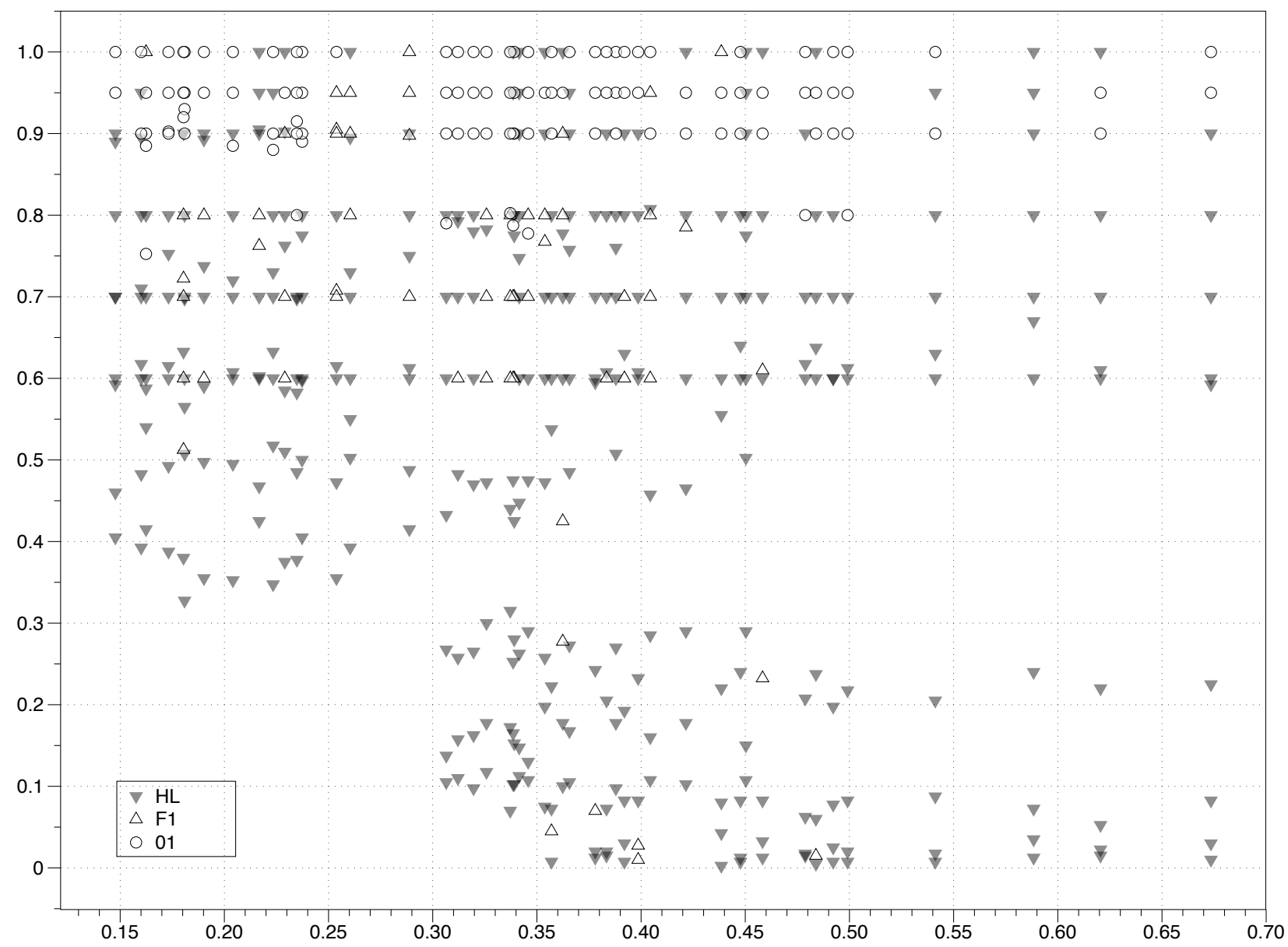

Fig. 4 Learners winning in $0 / 1$ loss in the 528 datasets. The horizontal axis represents the $0 / 1$ loss of datasets in the noise free releases. Thus points at the left hand side stand for easier datasets, typically those with less number of labels. The vertical axis represent the similarity of label sets, using $0 / 1$ similarity (9), with the noise free version. The higher the points in the figure, the lower the noise in the datasets 


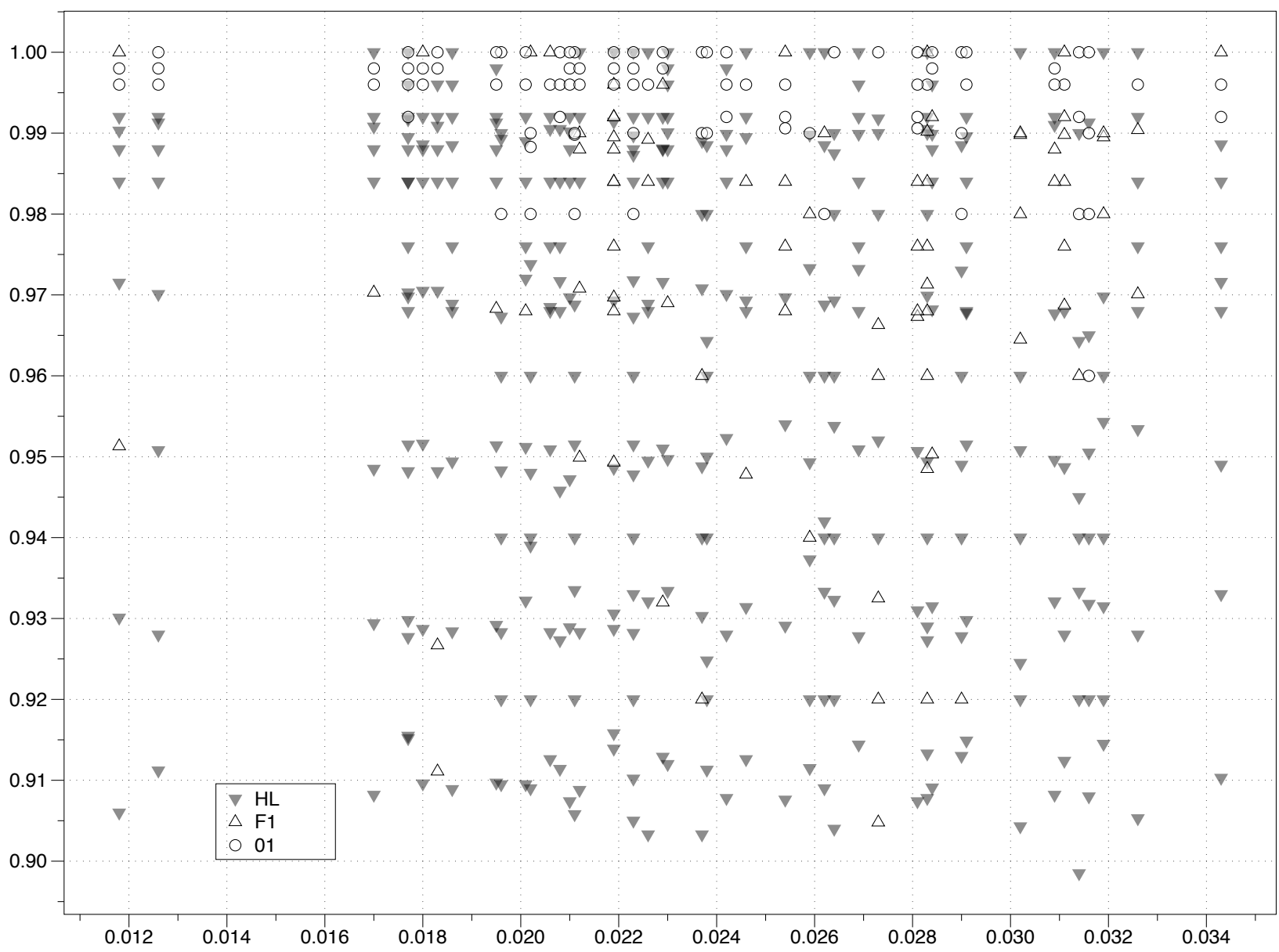

Fig. 5 Learners winning in Hamming loss in the 528 datasets. The horizontal axis represents the Hamming loss of datasets in the noise free releases. Thus points at the left hand side stand for easier datasets, typically those with less number of labels. The vertical axis represent the similarity of label sets, using Hamming loss similarity (9), with the noise free version. The higher the points in the figure, the lower the noise in the datasets 